\title{
Sistemas de medição de desempenho: uma revisão e classificação da literatura
}

\author{
Performance measurement systems: \\ a review and classification of literature
}

\author{
Rafael Tezza ${ }^{1}$ \\ Antonio Cezar Bornia ${ }^{1}$ \\ Ivan Henrique Vey ${ }^{1}$
}

\begin{abstract}
Resumo: O objetivo do presente artigo é classificar sistemas de medição de desempenho (SMD) de acordo com as abordagens utilizadas. Foram revisadas 140 abordagens sobre o tema, no período de 1980 a 2007 . Após a revisão, as abordagens foram classificadas baseando-se em cinco critérios: quanto à abrangência; quanto ao tipo de abordagem; quanto ao foco; quanto ao nível de medição; quanto ao grau de complexidade; bem como a algumas questões complementares. Verifica-se que o ambiente em que os SMD estão mais difundidos é o corporativo, no entanto, atualmente, várias abordagens vêm tratando da cadeia de suprimentos, serviços e indivíduo. A maioria das abordagens analisadas foi do tipo prática, com foco não financeiro e nos mais diversos níveis de medição e grau de complexidade. As contribuições deste trabalho concentram-se na geração de um melhor e sistematizado conhecimento sobre o tema de sistemas de medição de desempenho, bem como na identificação das tendências futuras dentro deste tema.
\end{abstract}

Palavras-chave: Sistemas de medição de desempenho. Revisão. Classificação.

\begin{abstract}
The purpose of this article is to classify Performance Measurement Systems (PMS) according to their approaches. In this paper 140 approaches were reviewed in the period 1980 to 2007. After the review, the approaches were classified on the basis of five criteria: on the scope, the type of approach, the focus, the level of measurement and on the degree of complexity. It appears that the environment in which PMS is most widespread is the corporate environment, however, several approaches are currently focused on the supply chain, services and individuals. Most of the approaches discussed were classified as practice, with no financial focus, and with different levels of measurement and degrees of complexity. The contributions of this work focus on generating a better and systematic knowledge on the subject of performance measurement systems and the identification of future trends within this topic.
\end{abstract}

Keywords: Performance Measurement Systems. Review. Classification.

\section{Introdução}

Antes mesmos da revolução industrial e do surgimento do paradigma fordista-taylorista, os sistemas de medidas de desempenho contábeis já eram utilizados pelos artesãos. No início do século XIX, os gerentes das fábricas tomavam decisões baseadas em informações sobre o custo por hora da transformação da matéria-prima em produtos acabados, particularizadas por departamentos e indivíduos. Desta forma, mediam a eficiência do processo e comparavam o desempenho produtivo de cada funcionário. Estas informações eram usadas para conceder remuneração adicional aos funcionários mais produtivos e estipular metas aos demais. Em meados do século XIX, os gerentes financeiros das ferrovias desenvolveram uma nova medida de desempenho: o índice operacional, que correlacionava o índice de despesas operacionais com a receita. Este índice era utilizado para medir a eficiência dos gerentes locais e a lucratividade dos negócios. As fábricas de produtos sob encomenda utilizavam essas informações produtivas e contábeis para determinar os produtos mais rentáveis e os preços de venda. Ainda no século XIX, Frederick W. Taylor fundou a administração científica, que substituía os métodos empíricos pelos científicos, por meio de procedimentos destinados a medir, de forma mais precisa, a quantidade de material, mão de obra e tempo de máquina necessários à produção (KAPLAN; COOPER, 1998).

No início do século XX, foi desenvolvido o indicador de retorno sobre o investimento (ROI - Return

\footnotetext{
${ }^{1}$ Programa de Pós Graduação em Engenharia de Produção, Universidade Federal de Santa Catarina, Campus Universitário - Trindade, CP 476, CEP 88040-900, Florianópolis - SC, Brasil, E-mails: rafaeltezza@ yahoo.com.br; cezar@inf.ufsc.br; ivanvey@ hotmail.com
} 
on Investiment), o qual, juntamente com outros indicadores financeiros, passou a ser empregado pelos decisores de grandes empresas para o controle da organização. Grande parte das organizações passou a adotar estes indicadores como único meio de avaliação de desempenho (JOHNSON; KAPLAN,1987).

A partir de 1960, com o movimento da qualidade, as empresas passaram a utilizar indicadores não financeiros focados em qualidade e em outras categorias internas, como a velocidade e a flexibilidade, ao lado do custo, como fatores importantes para o sucesso, fazendo com que os indicadores financeiros tradicionalmente utilizados se tornassem menos relevantes (BITITCI et al., 2001). Iniciava-se a ruptura com os indicadores puramente financeiros, reforçados a partir da década de 80 . Os estudos aprofundaram-se nos mais diversos tipos de abordagens: teóricos, práticos, financeiros, não financeiros, estratégicos, táticos, operacionais, focados em processo, pessoas e tantas outras variações.

Apesar desta grande variedade de abordagens, observa-se uma lacuna de estudos teóricos de análise e classificação a fim de embasar pesquisas sobre o tema, o que dificulta o planejamento de trabalhos acadêmicos. Considerando esta lacuna, o objetivo deste artigo é apresentar uma revisão da literatura das abordagens sobre medidas de desempenho desde o início da década de 1980 até 2007 e propor uma classificação para estas abordagens. Assim, este trabalho sistematiza os conhecimentos práticos e teóricos relacionados à área de SMD, classificando-os e identificando tendências, servindo de subsídio para futuros trabalhos teóricos e práticos. Para tanto, na seção 2 , são apresentados os procedimentos metodológicos utilizados. Na seção 3, apresentam-se os critérios utilizados para a classificação proposta. $\mathrm{Na}$ seção 4, é descrita a evolução dos SMD. Na seção 5, é apresentada e discutida a classificação das abordagens. Por fim, na seção 6, são feitas as conclusões finais.

\section{Procedimentos metodológicos}

Este trabalho se caracteriza quanto aos fins como exploratório, por se valer de uma busca por organizar conhecimentos acerca dos sistemas de medição de desempenho (SMD). Quanto aos meios, é uma pesquisa bibliográfica de estudos realizados sobre sistemas de medição de desempenho, publicados no período de 1980 até 2007.

O método de pesquisa utilizado para realizar o levantamento bibliográfico é apresentado na Figura 1.

No primeiro estágio, foram selecionadas as fontes iniciais, periódicos, anais de congressos, teses, dissertações e livros. Nesta etapa, realizou-se o levantamento no portal de periódicos da CAPES (http://www.periodicos.capes.gov.br/) das publicações relacionadas às áreas de Ciências Sociais Aplicadas, Ciências Sociais, Engenharias e Multidisciplinar. Foram pesquisadas 13 bases de dados: Emerald, Cambridge University Press, Gale, HighWire Press, ProQuest, Supply Chain Coaching, Elsevier, Ebsco, Scopus, Sage, SciELO, Science Direct e Wilson, além de livros e anais de congressos. No segundo estágio, para a seleção das referências, focou-se na busca por documentos que continham as seguintes palavras-chave no título e/ou no resumo: "Avaliação de Desempenho", "Gestão do Desempenho", "Mensuração do Desempenho", "Performance Assessment", "Performance Management", "Performance



Figura 1. Método de pesquisa bibliográfica utilizado. Adaptado de Villas et al. (2008). 
Measurement". A partir daí, foram analisados os títulos e os resumos dos artigos encontrados, selecionando-se os que apresentaram relevância para os objetivos do trabalho. O terceiro estágio - seleção de novos documentos - foi utilizado com o intuito de identificar, nas referências bibliográficas dos artigos encontrados no estágio 2 , as mais citadas, com o objetivo de inseri-las na classificação, tal como mostra a retroalimentação na Figura 1.

Após este processo, foram identificadas, como relevantes ao tema, 140 abordagens e os periódicos que representaram cerca de 54\% das abordagens aqui tratadas foram: International Journal of Operations \& Production Management; International Journal of Production Economics; Harvard Business Review; Management Accounting; Supply Chain Management Review; Accounting, Organizations and Society; Industrial Engineering; European Management Journal; International Journal of Production Research; European Journal of Operational Research.

A partir deste levantamento, foi desenvolvida uma classificação baseada nos critérios que apresentaram maior diferenciação entre as abordagens, seguindo a estrutura metodológica proposta por Godinho Filho e Fernandes (2003), que pode ser visualizada na Figura 2.

\section{Sistema de classificação proposto}

Os critérios utilizados na classificação da literatura pesquisada foram: abrangência, tipo de abordagem, foco, nível de medição e grau de complexidade.



Figura 2. Estrutura metodológica do trabalho. Fonte: Adaptado de Godinho Filho e Fernandes (2003).

\subsection{Abrangência da abordagem}

A primeira categoria da classificação proposta está relacionada à abrangência da abordagem e está vinculada ao estágio da cadeia produtiva em que o estudo foi realizado. Isto se justifica pelo fato de que os SMD, embora tenham surgido dentro das organizações e ligados diretamente aos processos, passaram a ser adotados e desenvolvidos nas mais diversas áreas. Esta categoria é subdividida: corporativa, cadeia de suprimentos, serviços e indivíduo. A abordagem corporativa está voltada às questões de estruturação geral da empresa (estratégias, relacionamento internos com clientes e fornecedores, organizações dos projetos, tratamento da informação etc.) e organização da produção e do trabalho (ligadas diretamente ao chão de fábrica). Segundo Rumler e Brache (1995), o desempenho de uma corporação pode ser visto em três níveis diferentes: o nível organizacional, o nível de processo e o nível executante. O nível organizacional coloca maior ênfase sobre as principais atividades funcionais da organização, enquanto o nível de processo centra-se no nível do fluxo de trabalho. Por fim, o nível executante é função da perspectiva de cada trabalhador.

A abordagem de medidas de desempenho em cadeia de suprimento está relacionada aos estudos referentes às interconexões entre empresas e o relacionamento existente entre elas, partindo de uma empresa focal e envolvendo todas as organizações que com ela se relacionam, desde a extração da matéria-prima até o cliente final, conforme definido pelo Council of Logistics Management.

Os estudos envolvendo a abordagem de serviços se fazem necessários, tendo em vista o significativo espaço que este setor vem assumindo. Para este trabalho, assume-se a definição de Miles (1996, p. 243), para o setor de serviços, que o classifica como "setor terciário que transforma o estado dos bens materiais, das próprias pessoas e dos símbolos (informação)".

As abordagens voltadas para o indivíduo vêm das análises de desempenhos individuais e são focadas nos recursos humanos, habilidades, treinamentos entre outros.

\subsection{Tipos de abordagem}

Esta categoria está relacionada com a metodologia do trabalho. Os procedimentos de pesquisa mais utilizados na área de Gestão da Produção, segundo Filippini (1997), são divididos em cinco classes: teórico-conceitual, experimental, pesquisa de avaliação (survey), estudo de caso e pesquisa-ação. Desta forma, a subdivisão quanto à abordagem, considerou dois subgrupos: abordagens teóricas e abordagens práticas. Na primeira, estão os estudos puramente teóricos, classificados como teórico-conceituais. Na segunda, 
estão os estudos que utilizaram de alguma forma a prática para validar algum conceito e são classificadas como experimental, pesquisa de avaliação (survey), estudo de caso ou pesquisa-ação.

\subsection{Foco}

O foco está relacionado com o caráter financeiro ou não financeiro. O caráter financeiro está ligado às abordagens que utilizam medidas puramente financeiras tais como lucratividade, custo de mão de obra, retorno sobre investimento, lote econômico e fluxo de caixa. As medidas não financeiras estão ligadas à qualidade, inovação, tempo de resposta, produtividade e flexibilidade.

\subsection{Nível de medição}

O nível de medição é subdividido em: estratégico, tático e operacional. O nível estratégico está ligado a abordagens focadas em aspectos vinculados ao futuro, que avaliam as estratégias subjacentes ao negócio e, como cita Anthony (1965), ligadas ao atingimento de objetivos macros, a longo prazo. O nível tático está ligado ao monitoramento das variações no desempenho operacional, mensuração de grupos de indivíduos ou processos e, ainda segundo Anthony (1965), relacionadas a controles administrativos e decisões sobre operações de controle. No nível operacional, segundo Anthony (1965), as ações resultam em uma resposta imediata, e as programações e procedimentos são estáveis, estando, desta forma, ligados diretamente ao feedback imediato e a medidas de rotina.

\subsection{Grau de complexidade}

Esta categoria está relacionada com alguns fatores estruturais das abordagens dos sistemas de medição, tais como, generalidade, quantidade de recursos envolvidos, qualificação dos executores, tecnologias envolvidas e sensibilidade às interpretações (avaliações qualitativas). Quanto maior o grau de utilização destes atores na abordagem, mais alta é a complexidade envolvida. Esta categoria foi subdividida em baixa complexidade, média complexidade e alta complexidade (Quadro 1).

\section{Evolução dos sistemas de medição de desempenho}

Esta seção expõe de forma resumida a maioria das abordagens sobre SMD pesquisadas. Discutindo a evolução destas desde 1980 até 2007.

Os estudos de Taylor no final do século XIX eram focados nas organizações e seus processos. Assim como as medidas de desempenho financeiras, que predominaram até o início dos anos de 1980.
Uma das primeiras abordagens não financeira e organizacional é a do 7-S da McKinsey (1980) desenvolvida no final dos anos 1970 nos EUA. Esta abordagem basicamente considera sete elementos organizacionais, subdivididos em objetivos (estratégia, estrutura e sistema) e subjetivos (habilidades, valor compartilhado, estilo e pessoas), de uma forma sistêmica e descritiva. Esta abordagem significou um avanço no sentido de considerar a organização de uma forma mais ampla e não puramente baseada em indicadores financeiros. Entretanto, como ressalta Petri (2005), pelo fato de ser uma abordagem puramente descritiva, mostra-se desfavorável no que diz respeito à competitividade e inovação. Em Watermann e Peters (1983), verifica-se uma tentativa de expandir a estrutura desenvolvida em 7-S da McKinsey (1980) inserindo agora o conceito de classe mundial. Nesta abordagem, os autores consideram os aspectos de eficiência organizacional e seus atributos para obter desempenho de "classe mundial". Os autores tentam romper com a ideia, vigente na época, de que os SMD tinham caráter punitivo. Eles também defendem a ideia de que não era mais suficiente a organização se conformar com o status quo, era preciso, segundo eles, se tornar criativo e inovador, servindo assim de complemento teórico para a abordagem de 7-S da McKinsey (1980). Neste período, são encontradas várias críticas aos SMD puramente financeiros, como é o caso de Kaplan (1983). Entretanto, ainda é possível encontrar, neste período abordagens puramente financeiras, como é o caso de Gold (1985) que aborda as medidas de desempenho sobre o ponto de vista de produtividade. Ele apresenta uma estrutura de relacionamento de custos focando basicamente a questão da rentabilidade de uma organização. A estrutura proposta por este autor apresenta três fatores:

Quadro 1. Codificação das subcategorias.

\begin{tabular}{|lcc|}
\hline \multicolumn{1}{|c}{ Categoria } & Subcategorias & Codificação \\
\hline Abrangência & Corporativo & CO \\
& Cadeia de suprimentos & CS \\
& Serviços & SE \\
Tipo de & Indivíduo & IN \\
abordagem & Teórico & TE \\
Foco & Prático & PR \\
& Financeiro & FI \\
Nível de & Não financeiro & NF \\
medição & Operacional & OP \\
& Tático & TA \\
Grau de & Estratégico & ES \\
complexidade & Baixo & BA \\
& Médio & ME \\
& Alto & AL \\
\hline
\end{tabular}


as relações de produtividade; os fatores de preço, custos unitários e perdas proporcionais.

Goberson (1985) é um dos primeiros trabalhos teóricos que descreve uma proposta de estágios para implementação de um conjunto de medidas de desempenho, apresenta técnicas para a escolha das medidas e descreve outras para a aferição de padrões destas medidas.

Neste período surgem abordagens mais especificas como é o caso de Sullivan (1986), uma abordagem puramente operacional, em que é desenvolvida a técnica OPTIM (Operating Profit Through Time and Investment Management) com o propósito de relacionar custo com outras dimensões de desempenho, traçando um perfil para avaliação e tomada de decisão.

Santori e Anderson (1987) afirmam que as medidas devem ser orientadas para o longo prazo, calculadas em base contínua no tempo, simples e de fácil entendimento, implementação e complementares entre si.

A tentativa de simplificação dos indicadores é o assunto de alguns autores como Son e Park (1987) e Keegan et al. (1989). Os primeiros propõem uma medida única para o desempenho da manufatura. Já Keegan et al. (1989) consideram um modelo simples que deveria acomodar qualquer medida necessária - a Matriz de Medição de Desempenho - que é baseado na inter-relação entre ambientes (interno e externo) e foca a questão custos.

Hiromoto (1988) compara diferenças das medidas financeiras adotadas em empresas dos EUA com as adotadas no Japão e conclui que no Japão a contabilidade gerencial tem o papel de ser influente em vez de simplesmente informar, como ocorre nos EUA.

No que diz respeito ao monitoramento do desempenho de processos tem-se as abordagens voltadas para o controle da qualidade total, que necessitam fortemente de indicadores de desempenho (JURAN, 1988; FORTUIN, 1988).

Na esfera estratégica, Baldrige Instituter (1988) faz uma abordagem que tem como objetivo identificar e ranquear as organizações dos setores de serviços e manufatura. Para tanto, busca identificar as boas práticas e seus resultados, fomentando a prática do benchmarking. Criando um conjunto de critérios para esta avaliação: Liderança; Planejamento Estratégico; Foco no Mercado e Clientes; Análise, Médias e Gestão de Conhecimentos; Foco nos Recursos Humanos; Processo de Gestão e Resultados (PETRI, 2005). No ano seguinte, Maskell (1989) trabalha o conceito Performance Measurement for World Class Manufacturer, um sistema de avaliação para empresas norte-americanas de classe mundial.

As abordagens puramente em serviços iniciaram-se primeiramente voltadas para a qualidade sob o ponto de vista do cliente, como é o caso do modelo SERVQUAL proposto por Parasuraman et al. (1988), no qual é proposto uma escala de 22 atributos divididos em cinco dimensões: elementos tangíveis, confiabilidade, capacidade de resposta, segurança e empatia. Brignall et al. (1991) propuseram um modelo de avaliação de desempenho também para o setor de serviços, baseado em 6 dimensões: competitividade, financeira, qualidade, flexibilidade, utilização de recursos e inovação. Os autores reforçam que estes fatores, determinantes do sucesso competitivo, devem ser mensurados e entendidos para que o sucesso da estratégia escolhida seja atingido.

Andersson et al. (1989) elaboram uma das primeiras abordagens voltada para a cadeia de suprimentos. Os autores elaboram uma proposta de medição que ilustra os aspectos quantificáveis do sistema logístico abordando medidas financeiras e não financeiras que os autores chamam de medidas físicas quantitativas.

A questão indivíduo na organização é aprofunda por autores como Zarifian (1990). A produtividade em atividades intelectuais, segundo ele, pode apresentar duas linhas de pesquisas distintas. Uma delas é tentar padronizar os métodos de trabalho intelectual e a outra seria baseada no conceito de atos de transação e não de operações intelectuais. Neste caso, a produtividade é medida pela velocidade de realização de um determinado número de transações. Senge (1990) propõe a ideia de modelos mentais com base em imagens, premissas e histórias que, segundo ele, têm efeitos diretos em nossa forma de agir e ver o mundo. Também discute o conceito de visão compartilhada, que é uma imagem futura compartilhada na qual possa existir envolvimento espontâneo dos funcionários. Aborda também o domínio pessoal, aprendizagem em equipe e pensamento sistêmico.

Voltando para a questão organizacional, ainda neste período, dentre as abordagens que tratam SMD e flexibilidade destaca-se a de Mishra e Pandey (1989) e Cross e Lynch (1990) - estes últimos criam o modelo SMART (Strategic Measurement and Reporting Tecnique).

Com um enfoque um pouco mais tático para identificar possíveis desvios, Dixon et al. (1990) propõem o Performance Measurement Questionnaire (PMQ) que é composto por 24 questões para diagnosticar a efetividade e os resultados dos SMD. Observa-se que neste período existe uma maior preocupação de ordem tático-operacional, que é reforçada em Azzone et al. (1991). Aqui os autores exploram a dimensão tempo, concentrando a escolha das medidas de desempenho em atividades críticas, tempo e custos. Defendendo assim a adoção de um pequeno número de indicadores. Grady (1991) retoma a ideia de "classe mundial", argumentando que as empresa devem dar ênfase ao benchmarking externo para definir seus objetivos, e demonstra exemplos de diagramação das lacunas (gaps). Mais tarde, Kasul e Motwani (1995) propõem um modelo estratégico que 
identifica o status de uma organização no caminho para o Desempenho de Manufatura Classe Mundial.

Campos (1992) insere na cultura ocidental a visão do relacionamento causa e efeito das atividades e, dentro deste conceito, baseado em um modelo japonês, propõe dois tipos de medidas: "Os itens de controle de um processo" e "Os itens de verificação de um processo".

Kaplan e Norton (1992) propõem o modelo Balanced Scorecard (BSC), uma estrutura complexa que envolve quatro perspectivas, uma financeira e três não financeiras (cliente, processo de negócio interno e da inovação e do aprendizado). Os autores sugerem, a partir destas perspectivas, uma estrutura que auxilia as organizações a traduzir as estratégias do negócio e a partir daí focar os esforços na gestão, no controle e na melhoria, utilizando conjuntos de medidas para as atividades mais críticas da organização. O BSC é, sem dúvida, o modelo de medição de desempenho mais difundido no mundo. A partir de sua divulgação, muitas abordagens têm sido publicadas, tanto abordagens como críticas ao modelo e também aplicações nas mais diversas áreas, como é o caso de Vokurka e Fliedner (1995), Heredia e Natarajan (1997), Drogelen e Cook (1997), Schneiderman (1999), Net Genesis Group (2000), Cravens et al. (2000), Brewer e Speh (2001), Figge et al. (2002) e Hubbard (2006), entre outros. Estas duas últimas abordagens representam uma adaptação do BSC voltada para a gestão ambiental e social, na qual são considerados os três pilares da sustentabilidade organizacional - econômica, ambiental e social. Esta adaptação é chamada pelos autores de SBSC (Sustainability Balanced Scorecard).

Dentro dos modelos desenvolvidos neste período, uma das espinhas dorsais é o processamento da informação, tema que chamou a atenção de autores como Kaydos (1991), Eccles (1991), Harrington (1993), Drucker (1995), Martins (2002) e Sharma e Bhagwat (2006) que se preocuparam com a confiabilidade das informações contidas nos SMD. Harrington (1993) estabelece três tipos de controle de processo: Eficácia, Eficiência e Adaptabilidade. Estas dimensões também são abordadas por Slack (1991) e Sink e Tutlle (1993). O primeiro toma as medidas de desempenho como uma vantagem competitiva baseada na adaptabilidade e sugere uma escala de nove pontos de desempenho, que estão diretamente ligados à percepção dos clientes e ao monitoramento dos concorrentes, construindo a partir daí uma matriz de importância/desempenho que evidencia as lacunas de desempenho e clarifica as prioridades do negócio. Sink e Tutlle (1993) propõem um modelo de avaliação de desempenho composto por sete critérios de desempenho: qualidade, eficiência, eficácia, inovação, qualidade de vida no trabalho, produtividade e lucratividade/ajuste ao orçamento. Em seus estudos os autores concluem que a avaliação ainda é muito mais percebida pelos usuários em geral como uma maneira de controle e de punição do que como uma ferramenta de apoio à tomada de decisão. Simons (1995) alerta que a pressão por resultados pode induzir as pessoas pressionadas a forjarem os números para demonstrarem bons resultados aparentes.

Barker (1993) retoma a questão tempo e propõe um sistema de medição de desempenho tomando como base o tempo para adicionamento de valor ao processo produtivo. Hendricks (1994), Åhlström e Karlsson (1996) e De Toni e Tonchia (1996), Maskell e Baggaley (2004) abordam as medidas de desempenho no modelo de produção enxuta. Em seus estudos, Åhlström e Karlsson (1996), concluem que os SMD tradicionais afetam negativamente as mudanças na estratégia de produção.

A avaliação de desempenho, voltada para os recursos humanos, ganha impulso com o modelo de avaliação focado no indivíduo chamado avaliação $360^{\circ}$ que é abordada por autores como London e Beatty (1993), Antonioni (1996) e Edwards e Ewen (1996). Neste modelo é proposta a utilização de múltiplas fontes, ou seja, a avaliação do empregado é realizada por diversos atores envolvidos no trabalho, como clientes, pares, chefe e subordinados. Fitz-Enz (1993), Hedges e Moss (1996), Kelly e Gennard (2001) e Gibb (2002) desenvolvem modelos para medição de desempenho no âmbito organizacional voltado para a área de recursos humanos como gestão de ativos para medir o desempenho de indivíduos e a relação custo benefício dos programas de formação.

Rummler e Brache (1994) avaliam o desempenho de organizações a partir de uma estrutura chamada de Abordagem dos Três Níveis, que leva em consideração nove aspectos para avaliar o desempenho e utiliza, assim como Lebas (1995), princípios da reengenharia. Lebas (1995) ressalta que a medição e a gestão do desempenho não podem ser separadas, o autor faz referência ao método de custeio ABC. Ostrenga et al. (1994) também definem medidas para todos os níveis organizacionais e definem três técnicas básicas para SMD: Identificar os fatores críticos de sucesso; Analisar um modelo de negócio baseado em processos; e Analisar causas básicas. A questão da identificação dos fatores críticos de Sucesso também é avaliada por Meyer (1994) que acrescenta mais sete técnicas: mapear os processos; selecionar as tarefas críticas e os fatores críticos a serem acompanhados; propor medidas de desempenho para as tarefas críticas e para os fatores críticos; definir as medidas de desempenho; testar as medidas de desempenho; submeter à revisão da gerência; e projetar o formato de relatório das medidas de desempenho.

Assim como o BSC de Kaplan e Norton (1992), outros modelos com alto grau de detalhamento e 
abrangência começam a surgir, como é o caso de Hronec (1994) que apresenta um modelo denominado modelo Quantum baseado em uma matriz que inclui três famílias de medidas: custo, qualidade e tempo. Este modelo apresenta uma visão abrangente do negócio, no entanto em contra partida necessita de muitos recursos para sua implementação.

A preocupação com a implementação dos SMD volta a ganhar espaço com os estudo práticos de Vitale et al. (1994) e Rose (1995). Neles são propostas sequências de passos para implementação de SMD priorizando o resultado final.

Neste período, várias ferramentas da gestão de qualidade começam a ser utilizadas em projetos de SMD, como apresentado em Bititci (1995), nos quais a estruturação de um indicador é descrita sob a forma de um diagrama de causa e efeito. $\mathrm{O}$ efeito está relacionado com a medida de desempenho de resultado e a causa está ligada ao processo que gera o resultado. O autor faz uso também da ferramenta QFD (Quality Function Deployment).

Para Lorino (1996), o desempenho é função do valor que as empresas oferecem aos clientes. $\mathrm{O}$ autor propõe uma rede de relacionamento entre as medidas de desempenho (financeira e não financeiras), pois, segundo ele, estas redes levam em conta as relações de causa e efeito existentes entre as medidas, utilizando como ferramenta para esta relação, o diagrama em árvore e sugere o uso dos "cinco por quês de Ohno" para construir tais diagramas. Daniels e Burns (1997), com base numa pesquisa-ação desenvolvida numa empresa de autopeças inglesa, propõem um sistema de medição de desempenho pró-ativo. A preocupação principal é o método de seleção, registro e uso das medidas selecionadas, e os fatores comportamentais envolvidos, mais que a seleção de medidas de desempenho tipo world class. O modelo é desenvolvido a partir do mapeamento das relações de causa e efeito cuja ligação é construída a partir do comportamento das pessoas. A ferramenta causa e efeito é abordada também por Juran (1988), Campos (1992) e Senge (1998).

Brown (1996) trabalha dois tipos de abordagem para a implementação de um sistema de medição de desempenho: abordagem top-down, recomendada a organizações com ênfase no controle e a abordagem unidade/localização, recomendada a organizações com mais autonomia. Neely et al. (1996) propõem um processo para desenvolvimento e implementação de SMD, considerando as necessidades do cliente e da empresa. A partir deste processo, os autores desenvolveram um workbook e uma folha de registro que foi testada com 200 gerentes.

Moreira (1996) apresenta seis dimensões do desempenho em manufatura e serviços: medida da utilização de recursos, da qualidade, do tempo, da flexibilidade, da produtividade e da capacidade de inovação. Para o autor, a seleção de indicadores de desempenho deve ser sustentada por quatro requisitos fundamentais: confiabilidade, validade, relevância e consistência. Griffin e Page (1996) argumentam em seus estudos que, para o sucesso financeiro, as medidas de desempenho mais utilizadas são: atendimento de metas de lucro e de metas marginais e o retorno sobre investimento.

Stewart (1995) retoma as abordagens na cadeia de suprimentos e alerta para a inadequação dos conceitos tradicionais de cadeia de suprimentos. Baseado em alguns estudos, propõe uma estrutura de indicadores balanceados para melhorar o desempenho da cadeia de suprimentos. Aponta quatro aspectos-chave para a excelência na cadeia de suprimentos: Desempenho da entrega; Flexibilidade e responsabilidade; Custo logístico; e Gerenciamento de ativos. Stainer (1997) coloca a produtividade, no âmbito das operações logísticas e mostra como ela pode ser medida pela análise de seu desempenho, propondo para isso um modelo para a gestão, destacando os determinantes a longo prazo para a eficácia. Christopher (1992) tem uma visão um pouco mais ampla da medição de desempenho na estrutura logística, ele aborda a medição e avaliação do desempenho sob duas perspectivas: em termos absolutos - baseado em custos; e em termos relativos - baseados na comparação com a concorrência (benchmarking). A perspectiva de custos é explorada também em Lambert et al. (1998), na qual os autores afirmam que o desempenho logístico pode ser monitorado pelas seguintes ferramentas: Padrões de custos e orçamentos flexíveis, Práticas orçamentárias, Padrões de produtividade e Controle estatístico do processo.

Boyd e Cox III (1997) fazem uso da Teoria das Restrições, em um estudo de caso, elaborando diagramas de relacionamento para expressar a relação, positivas e negativas, existente entre as medidas de desempenho.

No âmbito tático e estratégico, verificam-se abordagens como a da Universidade da Califórnia juntamente com o Departamento de Energia dos Estados Unidos (USA-DoE,1997), que desenvolveram uma abordagem para a administração total da qualidade e, para isso, métricas de desempenho que conduzam à avaliação quantitativa dos ganhos em: Satisfação do cliente; Desempenho organizacional; Excelência da força de trabalho. Gahalyini et al. (1997) desenvolveram o Sistema de Medição de Desempenho Integrado e Dinâmico (IDPMS), baseado na integração de três áreas funcionais: gerência, times de melhoria de processos e chão da fábrica. Três diferentes ferramentas ajudam as áreas funcionais a medirem e melhorarem de forma integrada: $o$ Performance Measurement Questionnaire, o conceito de meia vida e o diagrama do tempo de ciclo do valor modificado focalizado. 
Schalkwyk (1998) expõe dez diretrizes sobre a forma como os sistemas de medição de desempenho têm sido modificados em empresas que tenham implementado com sucesso TQM (Total Quality Management). Samson e Terziovski (1999) também analisam as práticas da TQM e o desempenho operacional de várias empresas com a finalidade de determinar a relação entre estas práticas e o desempenho individual e coletivo destas empresas.

Contextualizando o indivíduo dentro da organização, Guimarães (1998) sugere o desenvolvimento de um modelo integrado de gestão de desempenho, resultante de três etapas: planejamento, acompanhamento e avaliação. Estas duas últimas etapas formam, juntas, um mecanismo de retroalimentação (feedback) que, segundo a abordagem sistêmica, torna possível minimizar os efeitos da tendência à entropia a que as organizações estão sujeitas. No mesmo ano, Atkinson (1998) ilustra em um estudo de caso a forma como as medidas de desempenho estratégicas podem ser conduzidas por meio de uma hierarquia organizacional, fornecendo metas individuais que servem de base para planos de incentivos individuais. A estrutura de recompensas, atrelada aos indicadores de desempenho é também discutida por Santori e Anderson (1987), Eccles (1991), Vitale et al. (1994), Hronec (1994), Simons (1995), Cravens et al. (2000), Brewer e Speh (2001), Stank et al. (2001). Schiemann e Lingle (1999) abordam a cultura organizacional e apresentam um programa de desenvolvimento de uma cultura voltada para a medição de desempenho, que leva em conta quatro fases: definir; projetar; desdobrar; e sedimentar.

Waggoner et al. (1999), em uma revisão bibliográfica, determinam quais seriam as forças ambientais que modelam a evolução e mudança dos SMD. Identificaram quatro categorias de forças: Influências Internas; Influências Externas; Decisões de Processos; e Transformação.

As medidas de desempenho na cadeia de suprimentos voltam a ser abordadas em Beamon (1999). A utilização de um só indicador de desempenho - por exemplo, custo - é criticado, pois, segundo a autora, mesmo que uma cadeia de suprimentos opere com baixo custo, pode apresentar um fraco desempenho em outros aspectos como, por exemplo, o tempo de resposta ao cliente. Além das possíveis falhas na apuração dos custos totais. A autora estuda modelos quantitativos de avaliação de desempenho e sugere três tipos: Recursos (geralmente custos), Outputs (normalmente responsabilidade pelos clientes) e Flexibilidade (como $\mathrm{o}$ sistema reage à incerteza). A necessidade de existir correlação entre os indicadores de desempenho logístico e os indicadores de desempenho corporativo é defendida por Rey (1999), que cita quatro elementos de competitividade corporativa: Custos; Produtividade; Qualidade; e Tempo. A partir destes elementos estruturais, a autora sugere alguns critérios de decisão para a escolha dos indicadores, ligados a inter-relações e padronizações do processo.

Em Dreyer (2000), também se verifica a preocupação com a escolha dos indicadores. Para o autor, toda iniciativa de avaliação de desempenho deve se iniciar com um claro entendimento dos processos existentes. Isso é possível, segundo seus estudos realizados em uma planta da $G M$, com um mapeamento dos processos-chave da cadeia de suprimentos, que incluem: Obtenção; Aquisição; Processamento; e Distribuição.

Driva et al. (2000) concentram-se em estudar as medidas de desempenho utilizadas em pesquisa e desenvolvimento de produtos (PDP). Em um estudo prático envolvendo empresas de manufatura de todo o mundo, os autores propõem dez medidas de desempenho em PDP, que envolvem tempo, interpretação de requisitos, qualidade, falhas entre outros. A questão de medidas de desempenho em pesquisa e desenvolvimento é abordada também por Griffin e Page (1996), Durán (2001), Díaz et al. (2005) e Lin e Chen (2005).

Neely e Adams (2000) apresentam uma estrutura, multidimensional chamada Performance Prism que conta com cinco perspectivas: (i) satisfação dos stakeholders; (ii) as estratégias; (iii) os processos; (iv) os recursos; e (v) a contribuição dos stakeholders.

Bourne et al. (2000) trabalham no desenvolvimento e implementação de SMD e sugerem quatro processo para manter os SMD atualizados: (i) incluir um mecanismo efetivo para revisar e atualizar metas e padrões; (ii) incluir um processo para desenvolver novas medidas, assim que mudem as circunstâncias e o desempenho; (iii) incluir um processo para que, periodicamente, o conjunto completo de medidas empregadas seja revisado e alterado; e (iv) ser empregado para descobrir se as suposições estratégicas são válidas ou não.

Suwignjo et al. (2000) utilizam várias ferramentas para expressar relacionamentos entre fatores ou medidas de desempenho, entre elas estão os mapas cognitivos, diagramas de causa e efeito, diagramas árvore e a técnica do Processo Analítico Hierárquico (PAH). Com estas, os autores apresentam alguns benefícios, como possibilidade de redução do número de relatórios de medidas de desempenho, que se dá pelo maior entendimento das relações.

Ellinger e Daugherty (2000) mostram, em um estudo empírico, as relações entre a integração de marketing e logística interdepartamental no desempenho de uma empresa. Holmberg (2000) aborda a questão da troca de informação na cadeia de suprimentos. $\mathrm{O}$ autor afirma que a troca de informações pode auxiliar as empresas a desenvolver pensamento sistêmico em relação à cadeia de suprimentos e que os objetivos estratégicos devem ser definidos 
a partir das necessidades do cliente final da cadeia. Gunasekaran et al. (2001) têm uma abordagem semelhante, uma vez que propõem um modelo de avaliação de desempenho que mensura o que eles consideram os aspectos mais importantes de uma cadeia de suprimentos: Planejamento de ordens; Parcerias na cadeia; Produção; Distribuição; Serviço e satisfação do cliente; e Custos financeiros e logísticos da cadeia. Lambert e Pohlen (2001) propõem uma abordagem para o desenvolvimento de indicadores de desempenho para uma cadeia de suprimentos, alinhando o desempenho dos processos-chave entre pares de empresas, a partir das ligações existentes entre as empresas. As etapas da abordagem são: (i) Mapear a cadeia; (ii) Analisar cada ligação; (iii) Desenvolver uma demonstração do resultado para cada empresa; (iv) Realinhar processos da cadeia; (v) Alinhar indicadores não financeiros com financeiros; e (vi) Envolvimento dos parceiros.

A integração da cadeia de suprimentos e seus indicadores é um assunto relativamente recente, abordado por Dornier et al. (1998), Hoek (2001), Stank et al. (2001), Bowersox e Closs (1996) e Supply Chain Council (2002). Hoek (2001) aborda a garantia do desempenho de serviços à cadeia de abastecimentos por meio de mecanismos de controle horizontal.

Neste período, surgem várias abordagens particularizando alguns processos dominantes na época como em Devise e Pierreval (2000), que abordam indicadores de desempenho em sistemas de manufatura flexível, e em Fleury (2000), que faz uma abordagem dos sistemas de indicadores de desempenho no recente mercado de comércio eletrônico utilizando como estrutura o BSC. Kochhar e Zhang (2002) também estudam SMD em empresas virtuais, identificando que cada envolvido em um processo "virtual" e "não virtual" possui uma avaliação própria de desempenho, os autores então atentam para o sincronismo deste processo. Najmi e Kehoe (2001) fazem uma abordagem prática de medidas de desempenho em ambientes onde foi implantado ISO9000, pois segundo os autores existe uma falta de adequação dos SMD no pós-ISO9000. Robert (2000) e Searcy et al. (2007) discutem medidas de desempenho sob o ponto de vista de sustentabilidade organizacional, ou seja, consideram questões relacionadas com o meio ambiente e a sociedade. Segundo Robert (2000), os conceitos e ferramentas para medir o desenvolvimento sustentável - por exemplo, avaliação do ciclo de vida e pegada ecológica - devem medir o alinhamento das atividades com os princípios contidos em um quadro de sustentabilidade. Este, por sua vez, deve envolver a esfera econômica, social e ambiental, relacionando-se com o planejamento estratégico.

Algumas técnicas robustas são adaptadas por alguns autores para o desenvolvimento de SMD, como é o caso de Robinson et al. (2005) que discutem a implementação de indicadores-chave de desempenho (KPIs) em indústrias britânicas de construção civil. Outra técnica bastante estruturada e complexa utilizada para verificar desempenho, o DEA (Data Envelopment Analysis), é utilizada em um estudo de caso por Leachman et al. (2005) para avaliar as práticas de PDP, o tempo de produção e o grau de terceirização. Park e Park (2007) também utilizam esta ferramenta para avaliar a eficiência do serviço de uma empresa de televisão a cabo. Cliville et al. (2007) realizam um estudo de caso em uma empresa de médio porte e aplicam a ferramenta MacBeth de multicritério de apoio à decisão, um método bastante utilizado para quantificar elementos qualitativos de desempenho.

A medição de desempenho na economia digital, iniciada com Fleury (2000), Chin e Lee (2000) e Kochhar e Zhang (2002) ganha novas contribuições em Parasuraman et al. (2005), que evidencia a preocupação na avaliação, tanto na área corporativa quanto na de serviços, do fator humano e na interação deste com os sistemas de informação.

A cultura organizacional é o tema central das propostas de Bititci et al. (2005) e Henri (2006). Os primeiros tentam modelar a dinâmica de relacionamento entre SMD, estilo de gestão e cultura organizacional, com a finalidade de compreender melhor as relações causais entre as três. Henri (2006) aborda a relação entre cultura organizacional e SMD, considerando a influência desta no controle e na flexibilidade dos SMD.

Medidas de desempenho em serviços também são abordadas por Lee (2006). O autor faz um estudo de caso em escolas públicas na Malásia com o objetivo de avaliar empiricamente o quadro de desempenho da gestão pública, utilizando o BSC.

Sharma e Bhagwat (2006) desenvolvem uma estrutura que mede e avalia o desempenho dos sistemas de informação (SI) sobre seis perspectivas: a eficiência operacional da função do SI; baixo tempo do SI; responsividade do SI; a atualização da informação; precisão das informações; e a posição concorrencial global.

Ukko et al. (2007) avaliam os SMD na gestão e liderança de pessoas, os autores utilizam o BSC e destacam as diferentes perspectivas dos gestores e dos trabalhadores acerca da implementação dos SMD.

\section{Classificação e análise das abordagens}

A classificação das abordagens, quanto às cinco categorias apresentadas, pode ser verificada na Tabela 1, em ordem cronológica e alfabética dentro do ano de publicação. 
Tabela 1. Classificação das abordagens pesquisadas.

\begin{tabular}{|c|c|c|c|c|c|c|}
\hline \multirow[t]{2}{*}{ Abordagem } & \multirow[t]{2}{*}{ Ano } & \multicolumn{5}{|c|}{ Critério de classificação quanto à(ao) } \\
\hline & & Abrangência & $\begin{array}{c}\text { Tipo de } \\
\text { abordagem }\end{array}$ & Foco & $\begin{array}{l}\text { Nível de } \\
\text { medição }\end{array}$ & $\begin{array}{c}\text { Grau de } \\
\text { complexidade }\end{array}$ \\
\hline 7-S da McKinsey & 1980 & $\mathrm{CO}$ & PR & NF & TA & ME \\
\hline Watermann e Peters & 1983 & $\mathrm{CO}$ & $\mathrm{TE}$ & NF & ES & ME \\
\hline Kaplan & & $\mathrm{CO}$ & TE & FI, NF & TA, ES & ME \\
\hline Gold & 1985 & $\mathrm{CO}$ & $\mathrm{TE}$ & FI & $\mathrm{OP}$ & ME \\
\hline Globerson & & $\mathrm{CO}$ & $\mathrm{TE}$ & FI, NF & TA & BA \\
\hline Sullivan & 1986 & $\mathrm{CO}$ & PR & NF & $\mathrm{OP}$ & BA \\
\hline Son e Park & 1987 & $\mathrm{CO}$ & PR & NF & $\mathrm{TA}, \mathrm{OP}$ & BA \\
\hline Santori e Anderson & & $\mathrm{CO}$ & PR & FI, NF & ES, TA & BA \\
\hline Baldrige Instituter & 1988 & $\mathrm{CO}, \mathrm{SE}$ & PR & NF & ES & ME \\
\hline Hiromoto & & $\mathrm{CO}$ & PR & FI & TA, OP & BA \\
\hline Fortuin & & $\mathrm{CO}$ & $\mathrm{TE}$ & FI, NF & TA & BA \\
\hline Parasuraman et al. & & SE & $\mathrm{TE}$ & NF & ES & ME \\
\hline Juran & & $\mathrm{CO}$ & $\mathrm{TE}$ & FI,NF & TA,OP & BA \\
\hline Keegan et al. & 1989 & $\mathrm{CO}$ & $\mathrm{TE}$ & FI, NF & ES & $\mathrm{BA}$ \\
\hline Anderson e Storhagen & & $\mathrm{CS}$ & PR & FI, NF & ES & ME \\
\hline Maskell & & $\mathrm{CO}$ & PR & FI, NF & ES & ME \\
\hline Mishra e Pandey & & $\mathrm{CO}$ & PR & NF & TA, OP & BA \\
\hline Cross e Lynch & 1990 & $\mathrm{CO}$ & PR & FI, NF & ES, TA, OP & ME \\
\hline Crawford e Cox & & $\mathrm{CO}$ & PR & FI, NF & TA, OP & BA \\
\hline Zarifian & & IN & TE & NF & TA & ME \\
\hline Senge & & $\mathrm{CO}, \mathrm{IN}$ & $\mathrm{TE}$ & NF & ES & $\mathrm{AL}$ \\
\hline Dixon et al & & $\mathrm{CO}$ & PR & FI, NF & ES, TA, OP & ME \\
\hline Azzone et al. & 1991 & $\mathrm{CO}$ & $\mathrm{TE}$ & FI, NF & $\mathrm{TA}, \mathrm{OP}$ & BA \\
\hline Eccles & & $\mathrm{CO}$ & PR & FI, NF & ES & ME \\
\hline Grady & & $\mathrm{CO}$ & PR & FI, NF & ES, TA & ME \\
\hline Maskell & & $\mathrm{CO}$ & PR & FI, NF & TA, OP & BA \\
\hline Sink & & $\mathrm{CO}$ & $\mathrm{TE}$ & FI, NF & TA & ME \\
\hline Kaydos & & $\mathrm{CO}$ & $\mathrm{TE}$ & FI, NF & TA & ME \\
\hline Brignall et al. & & $\mathrm{SE}$ & PR & FI, NF & ES, TA & ME \\
\hline Slack & & $\mathrm{CO}$ & TE & FI, NF & TA,OP & ME \\
\hline Kaplan e Norton & 1992 & $\mathrm{CO}$ & $\mathrm{TE}$ & FI, NF & ES & $\mathrm{AL}$ \\
\hline Christopher & & $\mathrm{CS}$ & $\mathrm{TE}$ & FI, NF & ES, TA & ME \\
\hline Campos & & $\mathrm{CO}$ & $\mathrm{TE}$ & FI, NF & $\mathrm{OP}$ & BA \\
\hline Eccles e Pyburn & & $\mathrm{CO}$ & PR & FI, NF & TA & ME \\
\hline Harrington & 1993 & $\mathrm{CO}$ & $\mathrm{TE}$ & FI, NF & TA, OP & BA \\
\hline Fitz-Enz & & IN & PR & FI, NF & ES, TA & ME \\
\hline Barker & & $\mathrm{CO}$ & PR & FI, NF & $\mathrm{TA}, \mathrm{OP}$ & ME \\
\hline Sink e Tutlle & & $\mathrm{CO}$ & TE & FI, NF & $\mathrm{TA}, \mathrm{OP}$ & ME \\
\hline London e Beatty & & IN & PR & NF & TA & ME \\
\hline Hronec & 1994 & $\mathrm{CO}, \mathrm{IN}$ & PR & FI, NF & TA & $\mathrm{AL}$ \\
\hline Brown e Lecturer & & $\mathrm{CO}$ & PR & FI, NF & TA, OP & ME \\
\hline Hendricks & & $\mathrm{CO}$ & PR & NF & TA, OP & ME \\
\hline Rummler e Brache & & $\mathrm{CO}$ & PR & FI, NF & ES, TA, OP & ME \\
\hline Ostrenga e Orzan & & $\mathrm{CO}$ & TE & FI, NF & ES, TA, OP & ME \\
\hline Vitale et al. & & $\mathrm{CO}$ & PR & FI, NF & ES, TA & ME \\
\hline Meyer & & $\mathrm{CO}$ & PR & NF & $\mathrm{TA}$ & ME \\
\hline Neely et al. & & $\mathrm{CO}$ & TE & NF & TA & ME \\
\hline
\end{tabular}


Tabela 1. Continuação.

\begin{tabular}{|c|c|c|c|c|c|c|}
\hline \multirow[t]{2}{*}{ Abordagem } & \multirow[t]{2}{*}{ Ano } & \multicolumn{5}{|c|}{ Critério de classificação quanto à(ao) } \\
\hline & & Abrangência & $\begin{array}{c}\text { Tipo de } \\
\text { abordagem }\end{array}$ & Foco & $\begin{array}{l}\text { Nível de } \\
\text { medição }\end{array}$ & $\begin{array}{c}\text { Grau de } \\
\text { complexidade }\end{array}$ \\
\hline Bititci & 1995 & $\mathrm{CO}$ & PR & FI, NF & TA, OP & ME \\
\hline Drucker & & $\mathrm{CO}$ & $\mathrm{TE}$ & NF & ES & ME \\
\hline Griffin et al. & & $\mathrm{CO}$ & PR & NF & ES,TA & ME \\
\hline Rose & & $\mathrm{CO}$ & PR & NF & ES, TA & ME \\
\hline Kasul e Motwani & & $\mathrm{CO}$ & $\mathrm{TE}$ & NF & ES & ME \\
\hline Vokurka e Fliedner & & $\mathrm{CO}$ & PR & FI, NF & ES & $\mathrm{AL}$ \\
\hline Simons & & $\mathrm{CO}, \mathrm{IN}$ & $\mathrm{TE}$ & NF & TA & ME \\
\hline Lebas & & $\mathrm{CO}$ & $\mathrm{TE}$ & FI, NF & TA, OP & ME \\
\hline Stewart & & $\mathrm{CS}$ & PR & FI, NF & ES, TA & ME \\
\hline Edwards e Ewen & 1996 & IN & $\mathrm{TE}$ & NF & $\mathrm{TA}$ & ME \\
\hline De Toni e Tonchia & & $\mathrm{CO}$ & PR & FI, NF & TA, OP & ME \\
\hline Åhlström e Karlsson & & $\mathrm{CO}$ & PR & NF & TA, OP & ME \\
\hline Ghalayini e Noble & & $\mathrm{CO}$ & $\mathrm{TE}$ & FI, NF & ES, TA, OP & ME \\
\hline Moreira & & $\mathrm{CO}, \mathrm{SE}$ & $\mathrm{TE}$ & FI, NF & ES & ME \\
\hline Lorino & & $\mathrm{CO}$ & $\mathrm{TE}$ & FI, NF & TA, OP & ME \\
\hline Griffin e Page & & $\mathrm{CO}$ & PR & FI, NF & ES, TA & ME \\
\hline Flapper et al. & & $\mathrm{CO}$ & PR & FI, NF & TA, OP & ME \\
\hline Brown & & $\mathrm{CO}$ & $\mathrm{TE}$ & FI, NF & ES, TA & ME \\
\hline Bowersox e Closs & & $\mathrm{CS}$ & $\mathrm{TE}$ & FI, NF & TA, OP & ME \\
\hline Neely et al. & & $\mathrm{CO}$ & PR & FI, NF & TA & ME \\
\hline White & & $\mathrm{CO}$ & $\mathrm{TE}$ & FI, NF & ES, TA, OP & $\mathrm{AL}$ \\
\hline Antonioni & & IN & PR & NF & TA & ME \\
\hline Hedges e Moss & & IN & PR & NF & TA & ME \\
\hline Heredia e Natarajan & 1997 & $\mathrm{CO}$ & $\mathrm{TE}$ & FI, NF & ES, TA & ME \\
\hline Bititci et al. & & $\mathrm{CO}$ & $\mathrm{TE}$ & FI, NF & ES, TA, OP & ME \\
\hline Ghalayini et al. & & $\mathrm{CO}$ & PR & NF & ES,TA,OP & ME \\
\hline Daniels e Burns & & $\mathrm{CO}$ & PR & NF & $\mathrm{TA}, \mathrm{OP}$ & ME \\
\hline Sandia laboratories & & $\mathrm{CO}$ & PR & NF & TA,OP & $\mathrm{BA}$ \\
\hline Universty of California & & $\mathrm{CO}, \mathrm{SE}$ & PR & NF & ES,TA & ME \\
\hline Stainer & & CS & $\mathrm{TE}$ & NF & ES, TA, OP & ME \\
\hline Drogelen e Cook & & $\mathrm{CO}$ & $\mathrm{TE}$ & FI, NF & ES, TA & ME \\
\hline Boyd e Cox III & & $\mathrm{CO}$ & PR & FI, NF & TA, OP & ME \\
\hline Trade Family Nevada & & $\mathrm{CO}$ & $\mathrm{TE}$ & NF & $\mathrm{OP}$ & ME \\
\hline Schalkwyk & 1998 & $\mathrm{CO}$ & PR & $\mathrm{NF}$ & TA & ME \\
\hline Ballou & & $\mathrm{CS}$ & $\mathrm{TE}$ & FI, NF & TA, OP & ME \\
\hline Lambert et al.. & & $\mathrm{CS}$ & $\mathrm{TE}$ & FI, NF & TA, OP & ME \\
\hline Atkinson & & $\mathrm{CO}$ & PR & FI, NF & ES, TA & $\mathrm{ME}$ \\
\hline Dornier et al. & & $\mathrm{CS}$ & PR & FI, NF & ES, TA & ME \\
\hline Guimarães & & $\mathrm{CO}, \mathrm{IN}$ & $\mathrm{TE}$ & NF & ES, TA & ME \\
\hline Slack et al. & & $\mathrm{CO}$ & $\mathrm{TE}$ & FI,NF & ES,TA & ME \\
\hline Rey & 1999 & $\mathrm{CS}$ & $\mathrm{TE}$ & FI, NF & ES, TA & ME \\
\hline Beamon & & $\mathrm{CS}$ & $\mathrm{TE}$ & FI, NF & ES, TA & $\mathrm{AL}$ \\
\hline Schiemann e Lingle & & $\mathrm{CO}, \mathrm{IN}$ & PR & FI, NF & ES, TA & $\mathrm{AL}$ \\
\hline Waggoner et al. & & $\mathrm{CO}$ & $\mathrm{TE}$ & FI, NF & ES, TA & ME \\
\hline Samson e Terziovski & & $\mathrm{CO}$ & PR & NF & TA, OP & ME \\
\hline Cravens et al. & 2000 & $\mathrm{CS}$ & TE & FI, NF & ES & $\mathrm{AL}$ \\
\hline
\end{tabular}


Tabela 1. Continuação.

\begin{tabular}{|c|c|c|c|c|c|c|}
\hline \multirow[t]{2}{*}{ Abordagem } & \multirow[t]{2}{*}{ Ano } & \multicolumn{5}{|c|}{ Critério de classificação quanto à(ao) } \\
\hline & & Abrangência & $\begin{array}{c}\text { Tipo de } \\
\text { abordagem }\end{array}$ & Foco & $\begin{array}{l}\text { Nível de } \\
\text { medição }\end{array}$ & $\begin{array}{c}\text { Grau de } \\
\text { complexidade }\end{array}$ \\
\hline Bourne et al. & 2000 & $\mathrm{CO}$ & PR & FI, NF & ES, TA & ME \\
\hline Medori e Steeple & & $\mathrm{CO}$ & PR & FI, NF & ES & ME \\
\hline Driva et al. & & $\mathrm{CO}$ & PR & NF & ES, TA & ME \\
\hline Dreyer & & CS & PR & NF & ES, TA, OP & ME \\
\hline Suwignjo et al. & & $\mathrm{CO}$ & PR & FI, NF & ES, TA & $\mathrm{AL}$ \\
\hline Neely e Adams & & $\mathrm{CO}$ & $\mathrm{TE}$ & FI, NF & ES & $\mathrm{AL}$ \\
\hline Ellinger Daugherty & & $\mathrm{CS}$ & PR & NF & ES, TA & ME \\
\hline Fleury & & SE & PR & NF & ES, TA & ME \\
\hline Holmberg & & $\mathrm{CS}$ & PR & FI, NF & ES & ME \\
\hline Devise e Pierreval & & $\mathrm{CO}$ & $\mathrm{TE}$ & FI, NF & TA, OP & ME \\
\hline Robert & & $\mathrm{CO}$ & $\mathrm{TE}$ & FI, NF & ES & ME \\
\hline Net Genesis Group & & SE & $\mathrm{TE}$ & NF & ES, TA & $\mathrm{AL}$ \\
\hline Gunasekaran et al. & 2001 & $\mathrm{CS}$ & $\mathrm{TE}$ & FI, NF & ES, TA, OP & ME \\
\hline McAdam e Bannister & & $\mathrm{CO}$ & PR & NF & OP & BA \\
\hline Najmi e Kehoe & & $\mathrm{CO}$ & PR & NF & TA & BA \\
\hline Durán & & $\mathrm{CO}$ & $\mathrm{TE}$ & NF & $\mathrm{TA}$ & ME \\
\hline Brewer e Speh & & $\mathrm{CS}$ & PR & FI, NF & ES & $\mathrm{AL}$ \\
\hline Kelly e Gennard & & IN & $\mathrm{TE}$ & NF & TA, OP & ME \\
\hline Stank et al. & & $\mathrm{CS}$ & PR & FI, NF & TA, OP & ME \\
\hline Lambert e Pohlen & & $\mathrm{CS}$ & $\mathrm{TE}$ & FI, NF & TA & ME \\
\hline Hoek & & CS, SE & PR & NF & ES & ME \\
\hline De Toni e Tonchia & & $\mathrm{CO}$ & PR & FI, NF & TA, OP & ME \\
\hline Neely et al. & & $\mathrm{CO}$ & PR & FI, NF & ES & $\mathrm{AL}$ \\
\hline Kochhar e Zhang & 2002 & SE, CS & PR & NF & ES & ME \\
\hline Gibb & & IN & PR & NF & ES, TA & $\mathrm{AL}$ \\
\hline Martins & & $\mathrm{CO}$ & $\mathrm{TE}$ & FI, NF & ES & AL \\
\hline Figge et al. & & $\mathrm{CO}$ & $\mathrm{TE}$ & FI, NF & ES & $\mathrm{AL}$ \\
\hline Supply Chain Council & & $\mathrm{CS}$ & PR & FI, NF & ES, TA, OP & $\mathrm{AL}$ \\
\hline Ittner et al. & 2003 & $\mathrm{CO}$ & PR & FI, NF & ES, TA & ME \\
\hline Kennerley e Nelly & & $\mathrm{CO}$ & PR & NF & ES, TA & $\mathrm{AL}$ \\
\hline Maskell e Baggaley & 2004 & $\mathrm{CO}$ & PR & FI, NF & TA, OP & ME \\
\hline Lin e Chen & 2005 & $\mathrm{CO}$ & TE & FI, NF & ES & ME \\
\hline Bititci et al. & & $\mathrm{CO}, \mathrm{CS}, \mathrm{SE}$ & PR & NF & ES, TA & $\mathrm{AL}$ \\
\hline Robinson et al. & & $\mathrm{CO}$ & PR & FI, NF & ES & ME \\
\hline Parasuraman et al. & & SE & PR & NF & ES & ME \\
\hline Díaz et al. & & $\mathrm{CO}$ & PR & FI, NF & ES, TA & ME \\
\hline Leachman et al. & & $\mathrm{CO}$ & PR & FI, NF & ES, TA, OP & $\mathrm{AL}$ \\
\hline Bititci et al. & 2006 & $\mathrm{CO}$ & PR & $\mathrm{NF}$ & ES & ME \\
\hline Henri & & $\mathrm{CO}$ & PR & FI, NF & ES & $\mathrm{AL}$ \\
\hline Lee & & SE & PR & FI, NF & ES, TA & $\mathrm{AL}$ \\
\hline Hubbard & & $\mathrm{CO}$ & $\mathrm{TE}$ & FI, NF & ES & ME \\
\hline Sharma e Bhagwat & & $\mathrm{CO}$ & PR & NF & ES, TA & ME \\
\hline Park e Park & 2007 & SE & PR & FI, NF & ES & $\mathrm{AL}$ \\
\hline Ukko et al. & & $\mathrm{IN}, \mathrm{CO}$ & PR & NF & OP, ES & ME \\
\hline Searcy et al. & & $\mathrm{CO}$ & PR & NF & ES & ME \\
\hline Cliville et al. & & $\mathrm{CO}$ & PR & NF & ES & $\mathrm{AL}$ \\
\hline Wing et al. & & $\mathrm{CO}$ & PR & $\mathrm{NF}$ & ES & ME \\
\hline
\end{tabular}


Verifica-se que, a partir da década de 1980, houve um crescente número de abordagens sobre SMD, refletindo a preocupação com o tema. Na década de 1990, as publicações e as elaborações de modelos mostraram-se bastante densas; a partir dos anos 2000, a maioria das publicações constituiu-se de aplicações e adaptações dos modelos propostos nas décadas anteriores, por isso verifica-se uma redução no número de publicações consideradas.

A maioria das abordagens revisadas tem aplicação estritamente corporativa, como pode ser visualizado na Figura 3. Esta subclassificação é apresentada sobre uma perspectiva macro, considerando a abrangência corporativa, como descrito na seção 3 , como um universo que envolve a organização como um todo (estratégias, relacionamentos internos com clientes e fornecedores, organizações dos projetos, tratamento da informação, processos, entre outros). As demais categorias (Cadeia de Suprimento, Serviços e Indivíduo) foram encontradas em menor frequência, como já alertavam alguns autores, como Beamon (1999) e Gunasekaran et al. (2001). Entretanto, verifica-se, a partir de meados dos anos 1990, um aumento das abordagens tanto na categoria de Cadeia de Suprimentos como na categoria de Serviços e indivíduos, isoladamente ou em conjunto entre si, e também no âmbito corporativo. Este fato é justificado pelo recente interesse dado às relações da cadeia de suprimentos quando comparado com o dado ao ambiente corporativo e, mais recentemente, ao conceito de empresa estendida relaciona-se também a categoria de serviços.

A ocorrência de maior frequência de abordagens corporativas, provavelmente, se dá por dois motivos: primeiro, por ser uma abordagem bastante ampla e, segundo, por estar relacionada com a área em que historicamente se iniciaram os estudos de SMD.

Quanto ao tipo de abordagem, pode-se visualizar, na Figura 4, que nas duas primeiras décadas analisadas houve mais abordagens puramente teóricas, já a partir dos anos 2000 verifica-se maior número de abordagens práticas. Isto se dá pelo fato de que nas duas primeiras décadas houve mais desenvolvimentos de modelos, grande parte deles foi aplicada ainda naquele período, no entanto, a partir dos anos 2000, o que se verificou foram publicações de aplicações práticas de modelos já existentes, com pequenas adaptações.

Verifica-se que, ao longo do tempo, os três níveis de medição (operacional, tático e estratégico) receberam atenção. O que se pode observar é que, do final dos anos 1990 a 2007, a preocupação principal são as medidas de nível estratégico, sem é claro, deixar os outros dois níveis de lado (Figura 5). O que se pode deduzir é que o controle dos níveis operacionais e táticos é um pré-requisito básico para o sucesso organizacional ou de serviços.



Figura 3. Frequência das abordagens quanto ao tipo de abordagem nos períodos analisados.

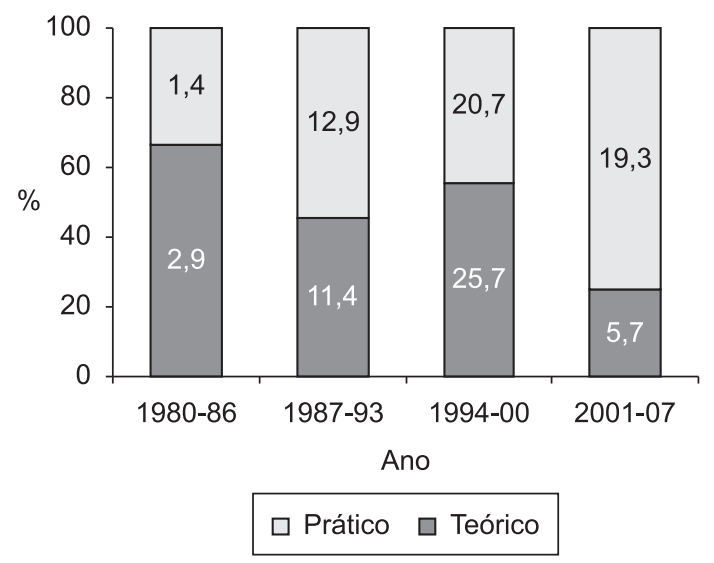

Figura 4. Frequência das abordagens quanto à abrangência nos períodos analisados. 




$\begin{array}{ll}\square \begin{array}{l}\text { Estratégico tático e } \\ \text { operacional }\end{array} & \square \text { Tático e operacional } \\ \square \text { Estratégico e tático } & \square \text { Estratégico } \\ \square \text { Tático } & \text { Operacional }\end{array}$

Figura 5. Frequência das abordagens quanto ao nível de medição nos períodos analisados.

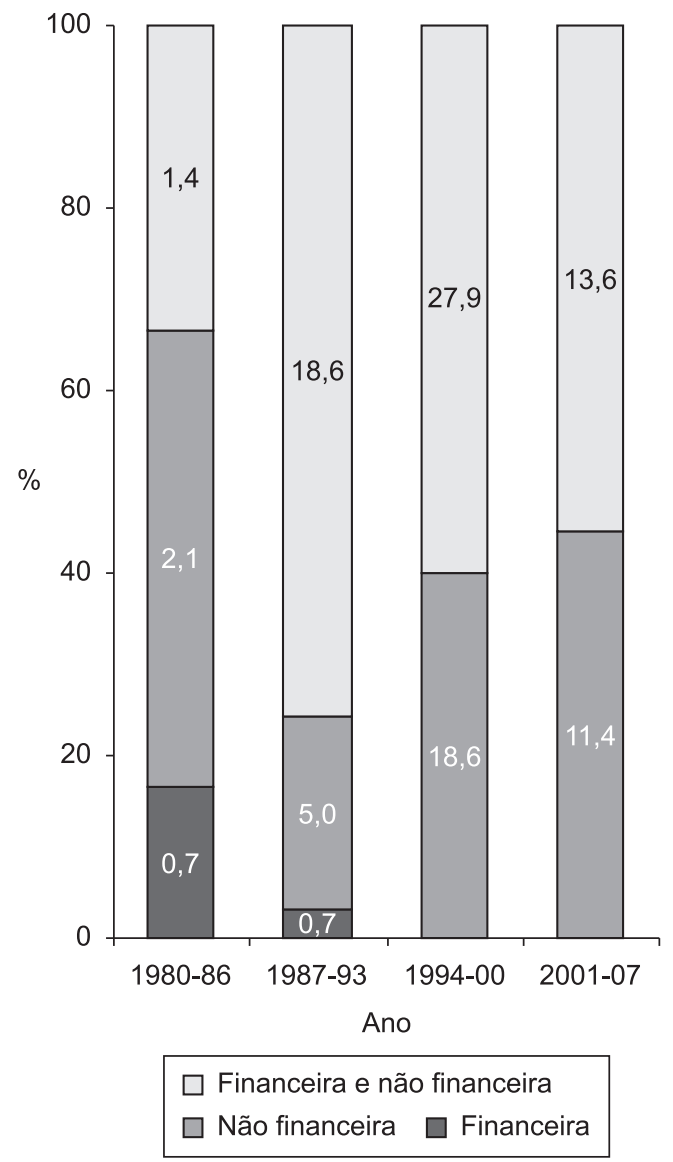

Figura 6. Frequência das abordagens quanto ao foco nos períodos analisados.

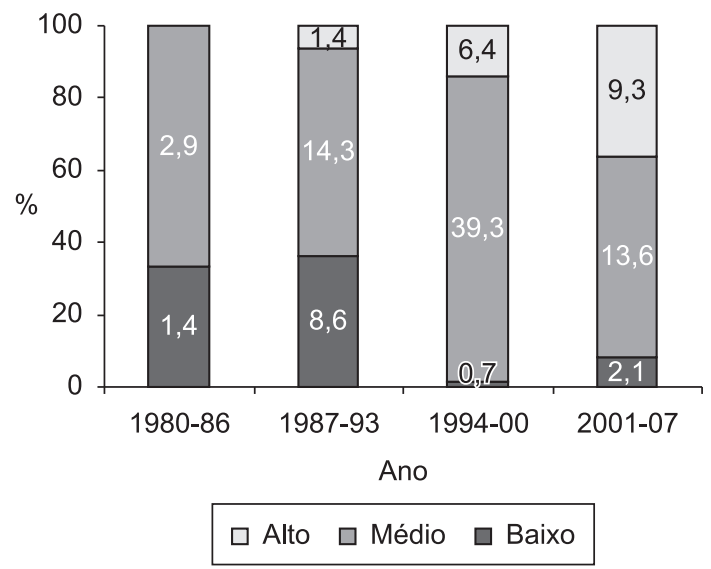

Figura 7. Frequência das abordagens quanto ao grau de complexidade nos períodos analisados.

A partir da década de 1960, quando começaram a surgir movimentos como o da qualidade e na sequência a gestão do conhecimento, as empresas passaram a dar mais ênfase às medidas de desempenho não financeira, o que teve sua abordagem intensificada na década de 1980 , como pode ser visualizado na Figura 6. Verifica-se que os modelos de medida de desempenho puramente financeiras, a partir da década de 1980 já eram menos difundidos que os puramente não financeiros e a associação de financeiros e não financeiros. E a partir de meados dos anos 1990, as abordagens puramente não financeiras e as associações financeiras e não financeiras são praticamente os únicos focos de estudo no âmbito de SMD.

Os modelos analisados, em sua grande maioria, possuem uma estrutura robusta e, na maioria das vezes, apresenta certo grau de complexidade, tanto em seu entendimento quanto em sua aplicabilidade, o que pode ser constatado na Figura 7, na qual se verifica que o grau de complexidade alto vem aumentando com o tempo, indicando que as modelagens estão envolvendo mais recursos, tecnologias, pessoas e fases do processo.

\section{Conclusão}

O levantamento bibliográfico apresentado cumpriu dois propósitos: o de ser abrangente e o de instigar futuras pesquisas. A classificação da literatura aqui apresentada corroborou para o aperfeiçoamento do assunto: Sistemas de Medição de Desempenho, possibilitando um direcionamento sobre alguns pontos de vista dentro deste tema, tais como abrangência das abordagens (corporativo, cadeia de suprimentos, serviços ou indivíduo), tipo de abordagem (prático e teórico), foco (financeiro, não financeiro), nível de abordagem (operacional, tático ou estratégico) e grau de complexidade de algumas abordagens. 
Observando-se a tendência das abordagens no tempo, percebe-se que o foco e a abrangência vêm evoluindo, abandonando o foco puramente financeiro - o qual não está presente nos trabalhos mais recentes - e surgindo abordagens com visão mais abrangente e voltada para o indivíduo e para fora das corporações a partir de meados da década de 1990 até $\mathrm{o}$ ano de 2007. A quantidade de abordagens de SMD na cadeia de suprimentos vem crescendo, o mesmo ocorrendo com a área de serviços, impulsionadas por modelos de terceirização. Observa-se que o grau de complexidade teve um aumento significativo nos últimos anos, acompanhando a complexidade dentro das organizações. Neste sentido, os esforços organizacionais para medir o desempenho têm sido visto pelos pesquisadores e práticos como um sistema completo, em vez de uma coleção de modelos autônomos e ferramentas.

De uma forma geral, os SMD são constituídos de alguns pontos-chave que são a confiabilidade das informações geradas, o mecanismo de causa e efeito e o impacto na cultura organizacional - diretamente ligada ao fator humano. Existe grande quantidade de abordagens nos mais diversos níveis, intensificando-se, com o passar do tempo, o nível estratégico.

Os trabalhos mais recentes têm evidenciado a influência do fator humano nos sistemas de medição de desempenho. Segundo Sinclair e Zairi (1995) e Gomes et al. (2004), a medição de desempenho tem impacto direto na motivação dos indivíduos envolvidos, o que obriga o administrador a priorizar o fator humano durante a elaboração dos SMD, tendo em mente que a responsabilidade adicional dos SMD relacionada com a atividade não deve vir à custa da produtividade e eficiência dos trabalhadores. Dentro deste contexto, verifica-se que começam a surgir abordagens preocupadas em estabelecer indicadores de desempenho social e ambiental (por exemplo, Robert (2000), Figge et al. (2002), Hubbard (2006) e Searcy (2007)).

Pela tendência observada, parece que as abordagens futuras dos SMDs manterão a abrangência da cadeia produtiva ou de serviço como um todo, sob a perspectiva da melhoria contínua, focando não apenas no projeto ou concepção de SMD, mas na aplicação e operacionalização destes, atentando para a complexidade envolvida na dinâmica organizacional.

O estudo apresentado representa um pequeno passo no sentido de compreender as filosofias envolvidas na elaboração e na aplicação de SMD bem como o direcionamento destes no atual ambiente de negócios. Entretanto, ainda existe muito a se avançar neste sentido. Uma possibilidade de pesquisa futura envolve a exploração de indicadores de desempenho sociais e ambientais e seus impactos nos resultados da organização, tanto em nível de eficiência interno quanto mercadológica. Outras pesquisas complementares seriam levantamentos da evolução dos SMDs utilizados na prática por empresas, a fim de se comparar as abordagens idealizadas na academia com a praxis empresarial.

\section{Agradecimento}

A pesquisa teve suporte parcial do CNPq. Os autores agradecem aos avaliadores anônimos pelas valiosas contribuições.

\section{Referências}

ÅHLSTRÖM, P.; KARLSSON, C. Change processes towards lean production: the role of the management accounting system. International Journal of Operations \& Production Management, v. 16, n. 1, p. 42-56, 1996.

ANDERSSON, P.; ARONSSON, H.; STORHAGEN, N. G. Measuring Logistics Performance. Engineering Costs and Production Economics, Vol. 17, p. 253-262, 1989.

ANTONIONI, D. Designing an effective 360-degree appraisal feedback process. Organizational Dynamics, v. 25, n. 2, p. 24-38, 1996.

ANTHONY, R. N. Planing and control systems: a framework for analysis. Cambridge: Harvard University Press, 1965. 180 p.

ATKINSON, A. Strategic performance measurement and incentive compensation. European Management Journal, v. 16, n. 5, p. 552-561, 1998.

AZZONE, G.; MASELLA, C.; BERTELÈ, U. Design of performance measures for time-based companies. International Journal of Operations \& Production Management, v. 11, n. 3, p. 77-85, 1991.

BALLOU, R. H. Business logistics management: planning, organizing, and controlling the supply chain. 4 ed. New York: Prentice Hall, 1998.

BARKER, B. Value-adding performance measurement: a time-based approach. International Journal of Operations \& Production Management, v. 13, n. 5, p. 33-40, 1993.

BEAMON, B. M. Measuring supply chain performance. International Journal Operations \& Production Management, v. 19, n. 3, p. 275-292, 1999.

BITITCI, U. S. Modelling of performance measurement systems in manufacturing enterprises. International Journal of Production Economics, v. 42, n. 2, p. 137-147, 1995.

BITITCI, U. S.; CARRIE, A. S.; MCDEVITT, L. Integrated performance measurement systems: a development guide. International Journal of Operations \& Production Management, v. 17, n. 5, p. 522-534, 1997.

BITITCI, U. S. et al. Measuring and managing performance in extended enterprises. International Journal of Operations \& Production Management, v. 25 n. 4, p. 333-353, 2005.

BITITCI, U. S. et al. Dynamics of performance measurement and organisational culture. International Journal of Operations \& Production Management, v. 26, n. 12, p. 1325-1350, 2006.

BOURNE, M. et al. Designing implementing and updating performance measurement systems. International 
Journal of Operations Management, v. 20, n. 7 , p. 754-771, 2000.

BOWERSOX, D. J.; CLOSS, D. C. Logistical management: the integrated supply chain process. New York: The McGraw-Hill Companies, 1996. (McGraw-Hill Series in Marketing)

BOYD, L. H.; COX, J. F. A cause-and-effect approach to analyzing performance measures. Production and Inventory Management Journal, v. 38, n. 3, p. 25-32, 1997.

BREWER, P. C.; SPEH, T. W. Adapting the balanced scorecard to supply chain management. Supply Chain Management Review, v. 5, n. 2, p. 48-56, 2001.

BRIGNALL, T. J. et al. Performance measurement in service businesses. Management Accounting, v. 69, n. 10, p. 34-36, 1991.

BROWN, D. M.; LECTURER, S. Measuring corporate performance. Long Range Planning, v. 27, n. 4, p. 89-98, 1994.

BROWN, M. G. Keeping score: using the right metrics to drive world-class performance. New York: Quality Resources, 1996.

CAMPOS, V. F. TQC: controle da qualidade total (no estilo japonês). Belo Horizonte: Fundação Cristiano Otoni, 1992.

CHIN, W. W.; LEE, M. K. O. On the formation of end-user computing satisfaction: a proposed model and measurement instrument. In: INTERNATIONAL CONFERENCE ON INFORMATION SYSTEMS, 21 ., 2000. Brisbane. Proceedings... Brisbane: Association for Information Systems, 2000. p. 553-563.

CHRISTOPHER, M. L. Logistics and supply chain management. London: Pitman Publishing, 1992.

CLIVILLE, V.; BERRAH, L.; MAURIS, G. Quantitative expression and aggregation of performance measurements based on the MACBETH multi-criteria method. International Journal Production Economics, v. 105, n. 1, p. 171-189, 2007.

CRAVENS, K.; PIERCY, N.; CRAVENS, D. Assessing the performance of strategic alliances: matching metrics to strategies. European Management Journal, v. 18, n. 5 , p. 529-541, 2000.

CRAWFORD, K. M.; COX, J. F. Designing performance measurement systems for just-in-time operations. International Journal of Production Research, v. 28, n. 1, p. 2025-2036, 1990.

CROSS, K. F.; LYNCH, R. L. Managing the corporate warriors. Quality Progress, v. 23, n. 4, p. 54-59, 1990.

DANIELS, R. C.; BURNS, N. D. A framework for proactive performance measurement system introduction. International Journal of Operations \& Production Management, v. 17, n. 1, p. 100-116, 1997.

DE TONI, A.; TONCHIA, S. Lean organization, management by process and performance measurement. International Journal of Operations \& Production Management, v. 16, n. 2, p. 221-236, 1996.

DE TONI, A.; TONCHIA, S. Performance measurement systems. International Journal of Operations \& Production Management, v. 21, n. 1-2, p. 46-70, 2001.

DEVISE O.; PIERREVAL, H. Indicators for measuring performances of morphology and material handling systems in flexible manufacturing systems. International
Journal Production Economics, v. 64, n. 1-3, p. 209-218, 2000.

DÍAZ, M. S.; GIL, M. J. Á.; MACHUCA, J. A. D. Performance measurement systems, competitive priorities, and advanced manufacturing technology. International Journal of Operations \& Production Management, v. 25, n. 8, p. 781-799, 2005.

DIXON J. R.; NANNI A. J.; VOLLMANN T. E. The new performance challenge: measuring operations for world class competition. Homewood: Irwin Professional Publishing, 1990.

DORNIER, P. P. et al. Global operations and logistics: text and cases. New York: Wiley, 1998.

DREYER, D. E. Performance measurement: a practitioner's perspective. Supply Chain Management Review, v. 4, n. 4, p. 63-68, 2000.

DRIVA, H.; PAWAR, K. S.; MENON, U. Measuring product development performance in manufacturing organizations. International Journal of Production Economics, v. 63, n. 2, p. 147-159, 2000.

DRUCKER, P. F. The information executives truly need. Harvard Business Review, v. 73, n. 1, p. 54-62, 1995.

DURÁN A. O. Sistemática para medição de desempenho em áreas de desempenho de produtos. In: CONGRESSO BRASILEIRO DE GESTÃO DE DESENVOLVIMENTO DE PRODUTO, 3., 2001, Florianópolis. Anais... Florianópolis: Universidade Federal de Santa Catarina, 2001.

ECCLES, R. G. The performance measurement manifesto. Harvard Business Review, v. 69, n. 1, p. 131-137, 1991.

ECCLES, R. G.; PYBURN, P. J. Creating a comprehensive system to measure performance. Management Accounting, v. 74, n. 4, p. 41-44, 1992.

EDWARDS, M. R.; EWEN, A. J. $\mathbf{3 6 0}^{\circ}$ Feedback: the new model for employee assessment \& perfomence improvement. New York: American Management Association, 1996.

ELLINGER, A. E.; DAUGHERTY, P. J. The relationship between marketing/logistics interdepartmental integration and performance in US manufacturing firms: an empirical study. Journal of Business Logistics, v. 21, n. 1, p. 1-22, 2000.

FIGGE, F. et al. The sustainability balanced scorecard: linking sustainability management to business strategy. Business Strategy and the Environment, v. 11, n. 5, p. 269-284, 2002.

FILIPPINI, R. Operations management research: some reflections on evolution, models and empirical studies in OM. International Journal of Operations and Production Management, v. 17, n. 7, p. 655-670, 1997.

FLAPPER, S. D. P.; FORTUIN, L.; TOOP, P. P. M Towards consistent performance management systems. International Journal of Operations \& Production Management, v. 16, n. 7, p. 27-37, 1996.

FLEURY, A. L. Um modelo de organização de negócios em mercados eletrônicos. Florianópolis, 2000. Dissertação (Mestrado) - Universidade Federal de Santa Catarina - UFSC.

FORTUIN, L. Performance indicators: why, where and how? European Journal of Operational Research, v. 34, n. 1, p. 1-9, 1988. 
GIBB, S. Learning and development: process, practices and perspectives at work. London: Palgrave, 2002.

GHALAYINI, A. M.; NOBLE, J. S. The changing basis of performance measurement. International Journal of Operations \& Production Management, v. 16, n. 8, p. 63-80, 1996.

GHALAYINI, A. M.; NOBLE, J. S.; CROWE, T. J. An integrated dynamic performance measurement system for improving manufacturing competitiveness. International Journal of Production Economics, v. 48, n. 3, p. 207-225, 1997.

GLOBERSON, S. Issues in developing a performance criteria system for in organization. International Journal of Production Research, v. 23, n. 4, p. 639-646, 1985.

GODINHO FILHO, M.; FERNANDES, F. C. F. Um sistema para classificar e codificar os trabalhos relacionados com o controle da produção e o controle da qualidade. Revista Gestão \& Produção, v. 10, n. 1, p. 89-107, 2003.

GOLD, B. Foundations of strategic planning for productivity improvement. Interfaces, v. 15, n. 3, p. 15-30, 1985.

GOMES, C. F.; YASIN, M. M.; LISBOA, J. V. A literature review of manufacturing performance measures. Journal of Manufacturing Technology Management, v. 15, n. 6, p. 511-530, 2004.

GRADY, M. W. Performance measurement: implementing strategy. Management Accounting, v. 73, n. 1, p. 49-53, 1991.

GRIFFIN, A. et al. Best practice for customer satisfaction in manufacturing firms. Sloan Management Review, v. 36, n. 2, p. 87-98, 1995.

GRIFFIN, A.; PAGE, A. L. PDMA success measurement project: recommended measures for product development success e failure. Journal of Product Innovation Management, v. 13, n. 6, p. 478-496, 1996.

GUIMARÃES, T. A. Gestão do desempenho em organizações públicas descentralizadas. In: CONGRESO INTERNACIONAL DEL CLAD, 3., 1998, Madri. Anais...

GUNASEKARAN, A.; PATEL, C.; TIRTIROGLU, E. Performance measures and metrics in a supply chain environment. International Journal of Operations \& Production Management, v. 21, n. 1-2, p. 71-87, 2001.

HARRIngton, H. J. Aperfeiçoando processos empresariais. São Paulo: Makron Books, 1993.

HENDRICKS, J. A. Performance measures for a JIT manufacturer: the role of IE. Industrial Engineering, v. 24, n. 1, p. 26-29, 1994.

HEREDIA, J. A.; NATARAJAN, R. A methodology framework for developing an integrated performance measurement system. In: RIBEIRA, J.; PRATS, M. J. (Eds.). Managing service operations: lessons from the service and the manufacturing sectors. Barcelona: University of Navarra, 1997. p. 59-64.

HUBBARD, G. Measuring sustainable organizational performance: win for you, win for me. Monash Business Review, v. 2, n. 3, p. 25-29, 2006

FITZ-ENZ, J. The mythology of measuring staff performance. Canadian Business Review, v. 20, n. 1, p. 17-20, 1993.

HENRI, J. F. Organizational culture and performance measurement systems. Accounting, Organizations and Society, v. 31, n. 1, p. 77-103, 2006.
HIROMOTO, T. Another hidden edge: japanese management accounting. Harvard Business Review, v. 66, n. 4, p. 22-26, 1988.

HOLMBERG, S. A systems perspective on supply chain measurements. International Journal of Physical Distribution \& Logistics Management, v. 30, n. 10, p. 847-868, 2000.

HRONEC, S. M. Sinais vitais. São Paulo: Makron Books, 1994.

ITTNER, C. D.; LARRCKER, D. F.; RANDALL, T. Performance implications of strategic performance measurement in financial services firms. Accounting, Organizations and Society, v. 28, n. 7-8, p. 715-741, 2003.

JOHNSON, H. T.; KAPLAN, R. S. Relevance lost: the rise and fall of management accounting. Boston: Harvard Business School Press, 1987.

JURAN, J. M. Planning for quality. New York: The Free Press, 1988. 341 p.

KAPLAN, R. S. Measuring manufacturing performance: a new challenge for managerial accounting research. The Accounting Review, v. 58, n. 4, p. 686-705, 1983.

KAPLAN, R. S. Yesterday's accounting undermines production. Harvard Business Review, v. 62, n. 4, p. 95-101, 1984.

KAPLAN, R. S.; COOPER, R. Cost and effect. Boston: Harvard Business School Press, 1998.

KAPLAN, R. S.; NORTON, D. P. The balanced scorecard: measures that drive performance. Harvard Business Review, v. 70, n. 1, p. 71-79, 1992.

KAPLAN, R. S.; NORTON, D. P. Putting the balanced scorecard to work. Harvard Business Review, v. 71, n. 5, p. 134-147, 1993.

KAPLAN, R. S.; NORTON, D. P. Using the balanced scorecard as a strategic management system. Harvard Business Review, v. 74, n. 1, p. 75-85, 1996.

KAYDOS, W. Measuring, managing and maximizing performance. Portland: Productivity Press, 1991.

KEEGAN, D. P.; EILER, R. G.; JONES, C. R. Are your performance measures obsoletes? Management Accounting, v. 70, n. 1, p. 45-50, 1989.

KELLY, J.; GENNARD, J. Power and Influence in the Boardroom. London: Routledge, 2001.

KENNERLEY, K.; NEELY, A. Measuring performance in a chaging business environment. International Journal of Production \& Operations Management, v. 23, n. 2, p. 213-229, 2003.

KOCHHAR, A.; ZHANG, Y. A framework for performance measurement in virtual enterprises. In: INTERNATIONAL WORKSHOP ON PERFORMANCE MEASUREMENT, 2., 2002, Hanover. Proceedings... Hanover: IFIP WG5.7, 2002. p. 2-11.

LAMBERT, D. M.; POHLEN, T. L. Supply chain metrics. The International Journal of Logistics Management, v. 12, n. 1, p. 1-19, 2001.

LAMBERT, D.; STOCK, J. R.; ELLRAM, L. M. Fundamentals of logistics management. Boston: McGraw-Hill, 1998.

LEACHMAN, C.; PEGELS, C. C.; SHIN, S. K. Manufacturing performance: evaluation and determinants. International Journal of Operations \& Production Management, v. 25, n. 9, p. 851-874, 2005. 
LEBAS, M. J. Performance measurement and performance management. International Journal of Production Economics, v. 41, n. 1-3, p. 23-35, 1995.

LEE, N. Measuring the performance of public sector organizations: a case study on public schools in Malaysia. Measuring Business Excellence, v. 10, n. 4, p. 50-64, 2006.

LIN, B.; CHEN, J. Corporate technology portfolios and R\&D performance measures: a study of technology intensive firms. R\&D Management, v. 35, n. 2, p. 157-170, 2005.

LONDON, M.; BEATTY, R. W. 360-degree feedback as a competitive advantage. Human Resource Management, v. 32, n. 2-3, p. 353-374, 1993.

LORINO, P. Le contrôle de gestion stratégique: la gestion par les activités. Paris: Dunod, 1996.

MARTINS, R. A. The use of performance measurement information as a driver in designing a performace measurement system. In: NEELY, A.; WALTERS, A.; AUSTIN, R. Performance measurement and management: research and action. In: PERFORMANCE MEASUREMENT AND MANAGEMENT CONFERENCE, 3., 2002, Boston. Proceedings... Boston: Performance Measurement Association, 2002. p. $371-378$

MASKELL, B. H. Performance measurement for world class manufacturing: a model for american companies. Portland: Productivity Press, 1991.

MASKELL, B. Performance measures of world class manufacturing. Management Accounting, v. 67, n. 5, p. 32-33, 1989.

MASKELL, B., BAGGALEY, B., Practical Lean Accounting, Productivity Press, New York, NY, 2004.

MCADAM, R.; BANNISTER, A. Business performance measurement and change management within a TQM framework A. International Journal of Operations \& Production Management, v. 21 n. 1-2, p. 88-107, 2001.

MEDORI D.; STEEPLE, D. A. framework for auditing and enhancing performance measurement systems. International Journal of Operations \& Production Management, v. 20, n. 5, p. 520-533, 2000.

MEYER, C. How the right measures help teams excel. Harvard Business Review, v. 72, n. 3, p. 95-103, 1994.

MILES, I. Innovation in services. In: DODGSON, M.; ROTHWELL, R. (Eds). The handbook of industrial innovation. Cheltenham: Edward Elgar, 1996.

MISHRA, P. K.; PANDEY, P. C. Simulation studies of flexible manufacturing systems using statistical design of experiments. Computers \& Industrial Engineering, v. 16, n. 1 , p. $65-74,1989$.

MOREIRA, D. A. Dimensão do desempenho em manufatura e serviço. São Paulo: Pioneira, 1996.

NAJMI, M.; KEHOE, D. F. The role of performance measurement systems in promoting quality development beyond ISO 9000. International Journal of Operations \& Production Management, v. 21, n. 1-2, p. 159-172, 2001.

NEELY, A.; ADAMS, C.; CROWE, P. The performance prism in practice. Measuring Business Excellence, v. 5 , n. 2, p. 6-12, 2001.
NEELY, A. et al. Realizing strategy through measurement. International Journal of Operations \& Production Management, v. 14, n. 3, p. 140-152, 1994.

NEELY, A. et al. Developing and testing a process for performance measurement system design. In: VOSS, C. A. (Ed.). Manufacturing strategy: operations strategy in a global context. London: London Business School, 1996. p. 471-476.

NEELY, A.; ADAMS, C. Perspectives on performance: the performance prism. Cranfield: University of Cranfield, 2000. Disponivel em: <http://www.inphase.com/ downloads/performance_prism.pdf $>$.

NET GENESIS GROUP. E-Metrics. Cambridge, 2000 Disponível em: <www.netgenesis.com/emetrics>. Acesso em: 12 de janeiro de 2008.

OSTRENGA, M. Guia da Ernst \& Young para a gestão total de custos. Rio de Janeiro: Record, 1994.

PARASURAMAN, A.; ZEITHAML, V. A.; BERRY, L. L. A conceptual model of service quality and its implications for future research. Journal of Marketing, v. 49, n. 4, p. 41-50, 1985.

PARASURAMAN, A.; ZEITHAML, V. A.; BERRY, L. L. SERVQUAL: multiple-item scale for measuring consumer perceptions of service quality. Journal of Retailing, v. 64, n. 1, p. 12-40, 1988.

PARASURAMAN, A.; ZEITHAML, V. A.; MALHOTRA, A. E-S-QUAL: a multiple-item scale for assessing electronic service quality. Journal of Service Research, v. 7, n. 3, p. 213-33, 2005

PARK, K. S.; PARK, K. Measurement of multiperiod aggregative efficiency. European Journal of Operational Research, v. 193, n. 2, p. 567-580, 2007.

PETRI, S. M. Modelo para apoiar a avaliação das abordagens de gestão de desempenho e sugerir aperfeiçoamentos: sob a ótica construtivista. Florianópolis, 2005. Tese (Doutorado) - Universidade Federal de Santa Catarina - UFSC.

REY, M. F. Indicadores de desempenho logístico. Revista Logmam, v. 30, n. 10, p. 86-90, 1999.

ROBERT, K. Tools and concepts for sustainable development how do they relate to a general framework for sustainable development, and to each other. Journal of Cleaner Production, v. 8, n. 3, p. 243-254, 2000.

ROBINSON, H. S. et al. Insights from research business performance measurement practices in construction engineering organizations. Measuring Business Excellence, v. 9, n. 1, p. 13-22, 2005.

ROSE, K. H. A performance measurement model. Quality Progress, v. 28, n. 2, p. 63-66, 1995.

RUMMLER, G. A.; BRACHE, A. P. Melhores desempenhos das empresas. São Paulo: Makron Books, 1994.

SAMSON, D.; TERZIOVSKI, M. The relationship between total quality management practices and operational performance. Journal of Operations Management v. 17, n. 4, p. 393-409, 1999.

SANTORI, P. R.; ANDERSON, A. D. Manufacturing performance in the 1990s: measuring for excellence. Journal of Accountancy, v. 164, n. 5, p. 141-147, 1987.

SCHNEIDERMAN, A. M. Why balanced scorecards fail. Journal of Strategic Performance Measurement, n. special edition, p. 6-11, 1999.

SEARCY C.; MCCARTNEY, D.; KARAPETROVIC, S. Sustainable development indicators for the transmission 
system of an electric utility. Corporate Social Responsibility and Environmental Management, v. 14, n. 3, p. 135-151, 2007.

SENGE, P. M. The fifth discipline: the art \& practice of the learning organization. New York: Doubleday \& Co., 1990

SHARMA, M. K.; BHAGWAT, R. Performance measurements in the implementation of information systems in small and medium-sized enterprises: a framework and empirical analysis. Measuring Business Excellence, v. 10 n. 4, p. 8-21, 2006.

SIMONS, R. Control in an age of empowerment. Harvard Business Review, v. 73, n. 2, p. 80-88, 1995.

SINCLAIR, D.; ZAIRI, M. Effective process management through performance measurement: part 1 . Business Process Re-engineering \& Management Journal, v. 1, n. 1, p. 75-88, 1995.

SINK, D. S. The role of measurement in achieving world class quality and productivity management. Industrial Engineering, v. 21, n. 6, p. 23-28, 1991.

SINK, D. S.; TUTTLE, T. C. Planning and measuring for performance. Rio de Janeiro: Quality Mark, 1993.

SLACK, N. The Manufacturing Advantage. London: Mercury Books, 1991.

SLACK, N. et al. Operations management instructors manual: operations management. London: Financial Times Prentice Hall, 1998.

SON, Y. H.; PARK, C. S. Economic measure of productivity, quality and flexibility in advanced manufacturing systems. Journal Manufacturing Systems, v. 6, n. 3, p. 193-207, 1987.

STAINER, A. Logistics: a productivity and performance perspective. Supply Chain Management, v. 2, n. 2, p. 53-62, 1997.

STANK, T. P. et al. Supply chain integration: tales from the trenches. Supply Chain Management Review, v. 5, n. 3, p. 62-69, 2001.

STEWART, G. Supply chain performance benchmarking study reveals keys to supply chain excellence. Logistics Information Management, v. 8, n. 2, p. 38-44, 1995.

SULLIVAN, E. OPTIM: linking cost, time, and quality. Quality Progress, v. 19, n. 4, p. 52-55, 1986.

SUWIGNJO, P.; BITITCI, U. S.; CARRIE, A. S. Quantitative models for performance measurement system. International Journal of Production Economics, v. 64, n. 1-3, p. 231-241, 2000.
UKKO, J.; TENHUNEN, J.; RANATANEN, H. Performance measurement impacts on management and leadership: Perspectives of management and employees. International Journal of Production Economics, v. 110, n. 1-2, p. 39-51, 2007.

USA-DoE (UNITED STATES DEPARTMENT OF ENERGY). How to measure performance: a handbook of techniques and tools prepared by the Training Resources and Data Exchange (TRADE) Performance-Based Management Special Interest Group (PBM-SIG). Washington, 1997.

VAN DROGELEN, I. C. K.; COOK, A. Measurements systems for $R \& D$ processes. R\&D Management, v. 27, n. 4, p. 345-357, 1997.

VAN HOEK, R. I. The contribution of performance measurement to the expansion of third party logistics alliances in the supply chain. International Journal of Operations \& Production Management, v. 21, n. 1, p. 15-29, 2001.

VAN SCHALKWYK, J. C. Total quality manangement and the performance measurement barrier. The TQM Magazine, v. 10, n. 10, p. 124-131, 1998.

VILLAS, M. V.; VAN ADUARD MACEDO-SOARES, T. D. L.; RUSSO, G. M. Bibliographical research method for business administration studies: a model based on scientific journal ranking. Brazilian Administration Review, v. 5, n. 2, p. 139-159, 2008.

VITALE, M.; MAVRINAC, S. C.; HAUSER, M. New process/financial scorecard: a strategic performance measurement system. Planning Review, v. 22, n. 4, p. 12-16, 1994.

VOKURKA, R.; FLIEDNER, G. Measuring operating performance: a specific case study. Production and Inventory Management Journal, v. 36, n. 1, p. 38-43, 1995.

WING, B. W. et al. Reducing conflict in balanced scorecard evaluations. Accounting, Organizations and Society, v. 32, n. 4-5, p. 363-377, 2007.

WAGGONER, D. B.; NEELY, A. D.; KENNERLEY, M. $P$. The forces that shape organizational performance measurement systems: an interdisciplinary review. International Journal of Production Economics, v. 60, n. 1, p. 53-60, 1999.

ZARIFIAN, P. As novas abordagens da produtividade. In: SOARES, R. M. S. N. Gestão da empresa, automação e competitividade: novos padrões de organização e relações de trabalho. Brasília: IPEA, 1990. 